Volume 8. No. 10, October 2020

International Journal of Emerging Trends in Engineering Research

Available Online at http://www.warse.org/IJETER/static/pdf/file/ijeter1328102020.pdf

https://doi.org/10.30534/ijeter/2020/1328102020

\title{
HIGH SPEED AND LOW POWER MULTIPLIERS DESIGNED USING REVERSIBLE LOGIC METHODS
}

\author{
Anitha $\mathbf{R}^{\mathbf{1}}$, Nithish Kumar $\mathbf{V}^{\mathbf{2}}$ \\ ${ }^{1}$ Assistant professor Senior, Vellore Institute of Technology, Vellore, TamilNadu, Email: ranitha@vit.ac.in \\ ${ }^{2}$ Associate Professor, Vellore Institute of Technology, Vellore, TamilNadu, Email: nithishkumarv@vit.ac.in
}

\begin{abstract}
Multiplier circuits take an important role in developing various digital gadgets, digital signal processing (DSP) and many other tasks. Multipliers are the main building blocks in conducting operations like convolution, filtering, etc. The research focuses on the nature of high-speed multipliers, decreased power consumption, and a lower region of low complexity. Recently, reversible logic has seen widespread use in the architecture of multipliers, low-power CMOS circuits, optical data processing, quantum circuits, etc. This paper uses reversible logic to construct compact multipliers using Vedic mathematics, Baugh-Wooley and Wallace tree architectures. In specific, the suggested multiplier designs are based on logics such as the reversible Half Adder, the reversible Full Adder, the Dual Key Gate and the Kogge Stone Adder. In order to ensure improved performance, a thorough distinction is made between different multipliers based on reversible logic circuits. The results of the proposed design are simulated and synthesized using the Xilinx ISE method.
\end{abstract}

Key words : Baugh Wooley Multiplier (BWM), Full Adder (FA ), Half adder (HA), Reversible Logic, Vedic mathematics, Xilinx ISE.

\section{INTRODUCTION}

The demand for high-speed multipliers has increased tremendously in recent years. In past literature several research efforts have been successfully carried out to design an energy-efficient multiplier [1-3]. Past studies have demonstrated that the circuits and systems designed in conventional design methods using irreversible logic results in higher power consumption and increased energy dissipation because of information loss [4]. The critical factor in the design and manufacturing of DSP devices for high-performance applications is low power usage and reduced delay [5-7]. The multiplier is a crucial circuit in a DSP application. The applications like Filtering (FIR, IIR), Fourier Transforms (FFT), convolution, etc. are depending on the multiplier circuits [8], [9], [10]. Multiplication is the most salient operations in almost all the computer processor, although it is more expensive and time-consuming [11, 12, and 13]. Multiplication can be carried out by different calculation problems that are regulated by speed [14]. Increased speed, reduced power and decreased area were observed using the multiplication process [15].

Reversible circuits are identical to standard circuits of logic except for gates that are implemented using reversible logic. There is a special one-to-one mapping of reversible gates with the inputs and outputs contradicting the traditional logic gates [16, 17]. Early work on reversible computing was carried out by R. Landauer's in early 1960s. If one bit of information is lost, the circuit dissipates heat in the form of energy which is at least $\mathrm{KT} \ln 2$ joules, where $\mathrm{K}$ and $\mathrm{T}$ are the Boltzmann constant and absolute temperature respectively [18]. The reversible logic gates are used to design the energy efficient circuits, and according to $\mathrm{C}$. $\mathrm{H}$. Bennett's research, reversible logic are capable of preventing KTln2 joules of energy dissipation in a circuit [19]. In [20], a $4 * 4$ parallel multiplier has been designed. The design included 3 steps viz. Partial Product Generation (PPG), Partial Product Dropping (PPD) and Addition of the decreased partial product produced in the previous steps. In [21], a new reversible gate called the HNG gate was proposed by the authors. Also the work [22] implemented the same technique as the two previous works, multiplying in two stages. Peres Full Adder Gate (PFAG) has been suggested by the researchers in this study. Also in this work, like the existing works, Peres gates were used to get the PPG. In [23], a new reversible gate named the RAM gate was introduced by the researchers. In [24], the system of Wallace Tree was planned to use two 
4-fold reversible multiplier circuits. The reversible multipliers were designed using the reversible gates like Feynman (FG), Peres (PG), Toffoli (TG), DKG and several other block operated as reversible half-adder and full-adder.

In this paper the half adders and full adders of the multiplier structure are replaced by the reversible logic gates. Instead of using a single reversible gate, here two different RG (Reversible gates) are used to design the adder. So that the garbage output is reduced, this in turn helps to reduce the delay and power consumed by the RG. All the existing design conceded with the garbage outputs, Ancillary inputs, quantum cost. But in this work, concentration has been focused on the parameters such as delay and power calculation. The proposed method has significantly reduced the depth of circuits and enhanced the speed. The delay and power in the proposed reversible multiplier design was very low compared with other existing circuit designs.

The rest of the paper is organized as follows: Section 2 briefs about the structure and its functionality of the basic reversible logic gates. The proposed reversible adder structure is described in the section III. The detailed architecture of various multipliers is studied in Section IV with the proposed Reversible Half Adder and Reversible Full Adder. Finally, the result has been discussed and analyzed with a conclusion of the proposed work.

\section{Structure and Functionality of Reversible Gates}

In general A (x, y) reversible logic gate has $\mathrm{x}$ number of inputs and $\mathrm{y}$ number of outputs. For example, $\quad$ A $(1,1)$ reversible logic is labeled as a gate for copying the inputs, i.e., the input goes unchanged through the gate. Meanwhile, there exists multiple input and output reversible gates and few are discussed below:

\subsection{NOT GATE}

It is the only reversible and irreversible logic gate [25], with input as A and output as A', which is shown in the below Figure.1

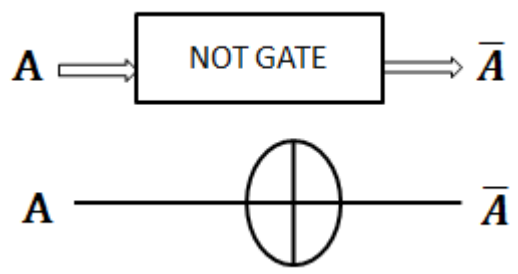

Figure. 1. Reversible NOT Gate

\subsection{FEYNMAN GATE}

With only $2 * 2$ Feynman gates [26] and converters, all reversible linear functions can be created. The gate of Feynman is called a CNOT gate (copying gate), with two inputs and two output logic gates. The logic function $\mathrm{W}=\mathrm{X}$ and $\mathrm{V}=X \oplus Y$ are shown in Figure.2. Feynman gate with an input as (X, Y) and output as $(\mathrm{W}, \mathrm{V})$.

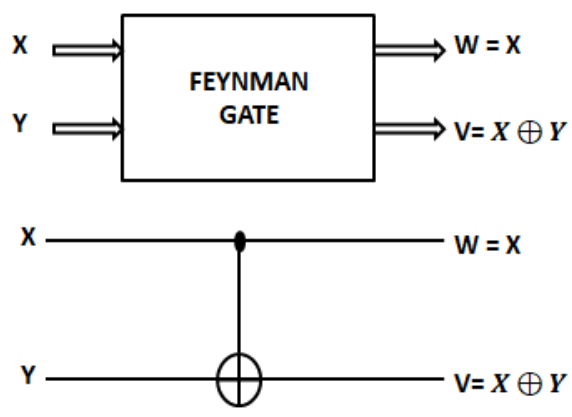

Figure. 2. FEYNMAN Gate

\subsection{TOFFOLI GATE}

The size of the Toffoli gate is incredibly small, which the most advantage of the gate. This logic gate has three inputs and three output [27]. The Toffoli portal is a "controlled-controlled-not gate" (CCNOT), in account of the two inputs ( $\mathrm{Y}$ and $\mathrm{Z}$ ) remain stable and when $\mathrm{B}=1$ and $\mathrm{C}=1$, the entrance inverts $A$. The logical function $\mathrm{W}=\mathrm{X}, \mathrm{V}=$ $\mathrm{Y}, \mathrm{U}=\mathrm{Z}^{\wedge}(\mathrm{XY})$, which is shown at Figure. 3 is, in Toffoli Gate with input as $(\mathrm{X}, \mathrm{Y}, \mathrm{Z})$ and output as $(\mathrm{W}, \mathrm{V}, \mathrm{U})$.
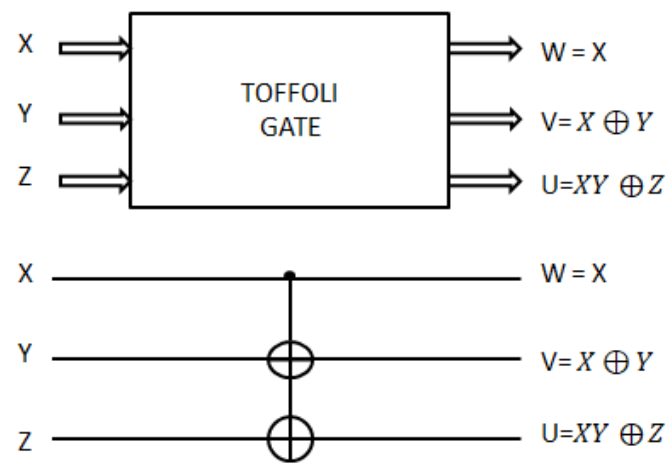

Figure. 3. TOFFOLI gate

\subsection{DUAL KEY GATE}

A $4 * 4$ reversible DKG gate will function as a FA and a full subtractor only as shown in Figure. 4a and 4b. If $\mathrm{A}=0$ is used, the DKG gate serves as a FA, while $A=1$ it will work as full Subtractor. To create special product combinations, a complete reversible circuit has been checked that at least 2 or 3 garbage outputs are required [26], [27]. 


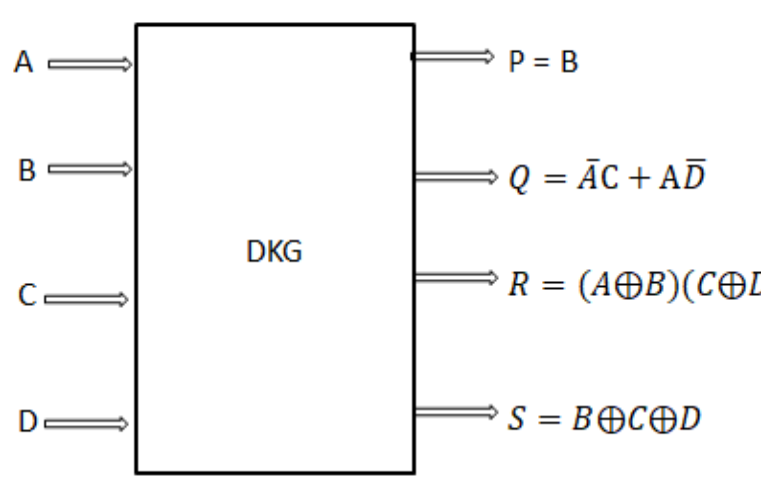

Figure. 4a. DKG gate

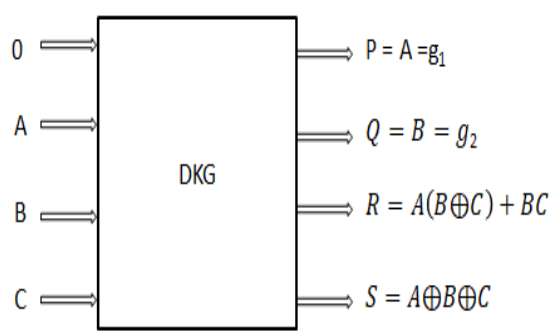

Figure. 4b. DKG gate as a Full adder

In the design of reversible logic gates, established design constraints have to be followed strictly or the constraints could be optimized for implementing any particular Boolean functions. It is essential that the number of inputs and outputs are retained equally in a reversible logic circuit. Similarly, each input pattern will have a specific output pattern. In a reversible logic circuit, fan out is not allowed as each output is used only once. Finally, the resulting reversible circuit should be an acyclic circuit. It must be taken care of some other considerations, the reversible circuits must use a less number of constant inputs, logic depth or gate rates, and the layout must be designed to minimize the number of garbage outputs. [28]. Toffoli gate and the Feynman Gates are used to design the Half Adder and Full Adder circuits which is discussed in the following sections.

\section{Proposed Reversible Adders}

In this section, several architectures of adders with reversible logic gates have been designed and are discussed as follows.

Approach I: Reversible Ripple Carry Adder (RCA) using DKG gate

From the discussion in the earlier section, the DKG gate can be used either as an adder or as a subtractor. Hence, in this work a reversible RCA using the DKG is designed which is shown in the Figure. 5. A $4 * 4$ DKG reversible logic gate that can operate only as a completely reversible adder or as a complete subtractor. It can be verified whether a common output pattern is uniquely specified input patterns. If the $A=0$ input acts as a reversible $D K G$ Full Adder gate and the $A=1$ input, then it acts as a DKG Full Subtractor Gate reversible. At least two waste outputs have been demonstrated to ensure the unique output combinations by the reversible full-adder circuit.

Approach II: Reversible Ripple Carry Adder using Feynman and Toffoli gates

Similarly, in the next approach the Reversible Half Adder (RHA) is designed using one Toffoli gate and one Feynman Gate which is shown in Figure 6. As per the logic, the single full adder performs addition of two 1-bit numbers and a carry input. Also, a reversible full adder (RFA) can be designed using two RHA and one OR gate as shown in Figure. 7a. In order to perform addition of numbers with more than 1-bit, parallel adders like RCA structure are designed using reversible full adder (RFA). Figure $7 \mathrm{a}$ and $7 \mathrm{~b}$ shows the reversible full adder and Reversible RCA. The propagation delay in RCA is very high but by using the RHA and RFA the delay is getting reduced and power consumed by the reversible logic gates are very less because of switching of inputs, when compare to the logic gates.

Approach III: Reversible Kogge Stone Adder using RHA and RFA

Because of the complexity $\mathrm{O}(\log 2 \mathrm{~N})$ delay along the carry route compared to other adders, the parallel-prefix tree adders are more desirable in terms of size. The literature therefore considers that the Kogge-stone adder is the fastest adder in comparison with other additions. The adder choice in terms of worst-case delay is seen by Ripple-Carry, Carry-Look-Ahead, Carry-Select, and Kogge-Stone which are few famous parallel prefix adders. This is related to the "Reduced number of Stages". The implementation of KSA is very simple and uncomplicated structure and among all the other carries tree adders and it has the shortest critical path. Due to that there is a decrease in delay to generate the carry. But it consume larger area and routing of interconnects will be a complex one, which both are the disadvantage of KSA. Hence KSA is used for fastest arithmetic functions which are popularly used in DSP, Control Systems etc.

The RHA and RFA which has the ability to reduce the power which discussed in the above approach II, so the adder circuit of KSA is redesigned by using this to achieve the low power and high speed outputs 
when compare to other tree adders.
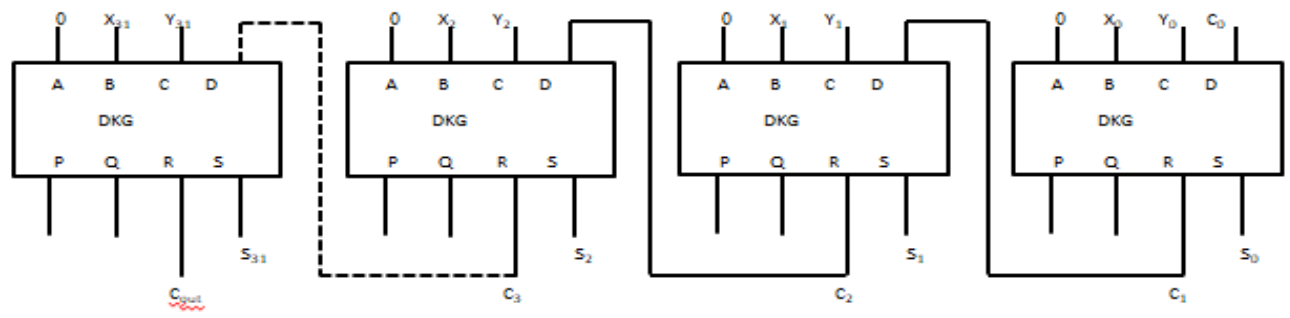

Figure. 5. 32-bit Reversible RCA using DKG.

\section{Proposed Multiplier Architecture using THE REVERSIBLE ADDER}

DSP operations such as convolution, discrete transformation wavelet, Fast-Fourier transformation and filtration, etc., the multipliers are the basic building blocks. The device speed is determined by the computation time of the multiplication operation. The Vedic approach for multiplication is one of the best places for the faster operation, by removing the unnecessary steps in the process of multiplication. Efficient adders should be chosen when designing the multipliers so that the multipliers have less delay and becomes power efficient. Similarly, the binary numbers multiplication problem should be resolved using similar methods to improve the consistency of the current aphorism with modern systems.

\section{1 Vedic Urdhva Tiryakbhayam Multiplier}

Vedic math is an ancient mathematics have the sutra (aphorisms), including arithmetic, algebra, geometry, and so many mathematic functions. The term 'Vedic' comes from the term 'Veda' which is the house of power and divinity. [29,30]. The Vedic multiplier suggested is based on sutra (algorithm), "Urdhava Triyakbhayam." These Sutras were traditionally used in the decimal number system to subtract two numbers. Urdhava Triyakbhayam (UT) Sutra means "Vertically and Crosswise". Shifting operation is not required in UT, as partial product measurement is carried out in one stage, saving time and power in turn. The Vedic multiplier has this huge benefit which is shown in Figure. 8[31]. A 32-bit UT multiplier is design with three methods, in all designs the RCA was replaced by the proposed reversible adders as shown in Figure. 8

\section{2 Baugh Wooley Multiplier}

If the signed $\mathrm{n} * \mathrm{n}$ multiplication is done, if the output has a bit width equal to the inputs then there won't be any difference in the design. The effective algorithm in the multiplier to deal with this situation is BWM. In order to design structured multipliers suitable for the 2 supplementary numbers [30], this design method was established. By extracting the last two positive terms from the first two positives, it will generate the final output product. Instantly, the two can be applied to the last two words and adding both terms to deliver the final result instead of subtracting as normal multipliers.

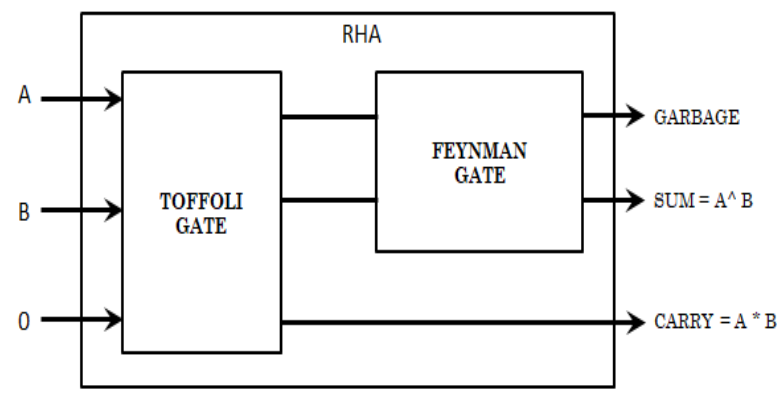

Figure.6. Proposed Reversible Half Adder (RHA) using Toffoli

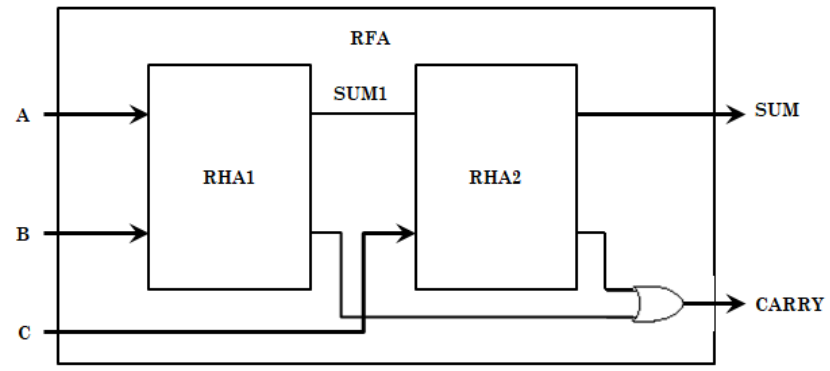

Figure. 7a. Proposed Reversible Full Adder (RFA) using RHA

Reversible BWM:

The BW multiplier is the efficient to handle signed bit The 2's complement number can be easily handled by the BWM. In the paper [33], the reversible multiplier is designed using Reversible TSG gate as a full adder and FRG gate for the AND gate and compared the 
delay and power. Likewise, the reversible logic gates are utilized to design the Baugh Wooley Multiplier and analyzed the power and delay with the existing Baugh Wooley Multiplier. The following Figure. 9 shows the block diagram of $32 * 32$ BMW. In this Baugh-Wooley, there are two different blocks, one is grey block and other is white box. These two boxes the grey and white implementation which is implemented using proposed RFA and RHA (i.e. method II) as shown in Figure. 9.

Similarly, the RCA in the last stage of BWM is replaced by the proposed Adders from Method I to method III as shown in the Figure. 9. The power and delay is calculated for the each and every method and the result is shown in the below table 1 .

\section{3 Wallace Tree Multiplier}

Another high speed multiplier is the Wallace Tree Multiplier, and which consumes less power among all the other tree multipliers. There are two structures in the Wallace tree multiplier which are conventional multiplier and reduced complexity multiplier, here conventional multiplier is considering for the design. An assortment of number-crunching procedures has been introduced to plan compelling multiplier. Probably the best strategy is the Wallace tree method as shown in Figure.10. This approach allows efficient equipment containing full and half-adder circuits to be generated in three phases that similarly influence the rise in operation. The Wallace-based duplicating activity incorporates three phases [34] as follows

Satge 1: Multiply each multiplier (or better express AND) bit, and produce n2partial products by each multiplier bit.

Satge 2: Lower the number of PP with the Full and Half Adder block layers.

Satge 3: Two n-sets have been added to an n-bit adder from the previous step.

Similarly, the Adders (HA \&FA), conventional Adder of the Wallace tree multiplier shown in Figure. 10 is replaced with DKG reversible gate which is described in the method 1 , secondly it has been designed using the method 2 which is RFA, RHA and Reversible RCA and finally the last stage of the Wallace tree multiplier is designed and implemented using the proposed RKSA. The Power and Delay of all the three methods have been calculated using the Xilinx Tool. The Result is compared with the existing method in which is given in the paper [24].

\section{RESULT ANALYSIS AND SYNTHESIS}

The implementations of the proposed methods are designed using Verilog HDL. The comparison of delay and power of $32 * 32$ of the proposed multipliers and reversible array multiplier using Xilinx ISE tool with the Virtex 5 device is shown in table 1. The reversible multiplier architectures are designed and implemented using Xilinx ISE tool to calculate the delay. Xpower analyze tool is used to calculate the power.

\section{CONCLUSION}

In this paper, the reversible multiplier architectures based on different techniques like Vedic mathematics, Baugh Wooley and Wallace tree methods are designed, simulated and analyzed. The reversible multipliers are extensively used to build higher order multipliers, DSP applications and complex circuits like quantum computers. The performance of the different multiplier structures are evaluated by measuring the parameters like delay and power. In terms of delay, Vedic Multiplier with Proposed RKSA has recorded the best performance with lowest delay and lowest power consumption. Wallace tree architectures based on RHA and RFA as well as DKG based design has also produced best results in terms of delay and power consumption. The proposed multiplier structures are implemented in hardware using Xilinx Virtex 5. As a future work, the area requirements, number of gates, garbage outputs for each of the reversible multiplier structure will be computed and the results are analyzed. 
Anitha R et al., International Journal of Emerging Trends in Engineering Research, 8(10), October 2020, 7491 - 7500

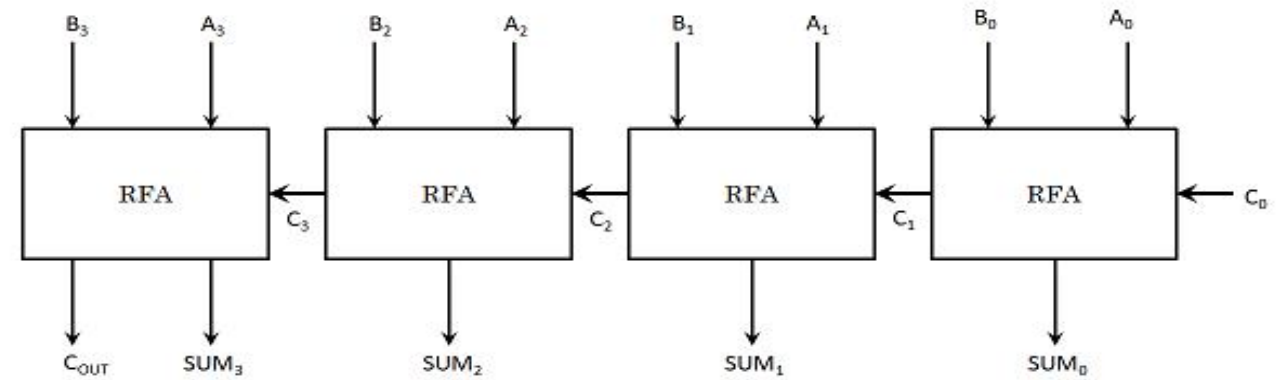

Figure.7b. Proposed Reversible RCA using proposed RFA

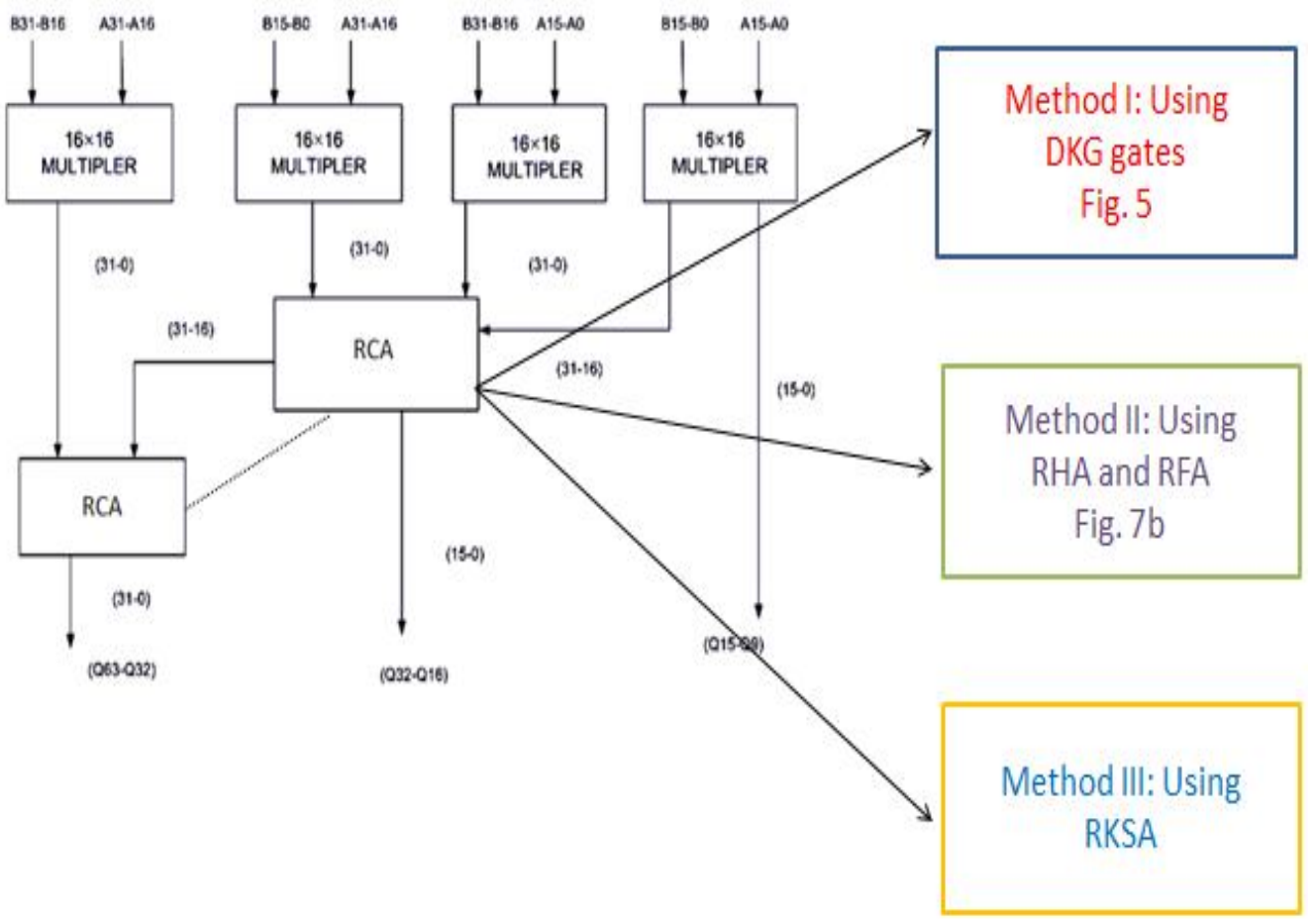

Figure. 8. 32- bit Urdhva Tiryakbhayam Sutra 


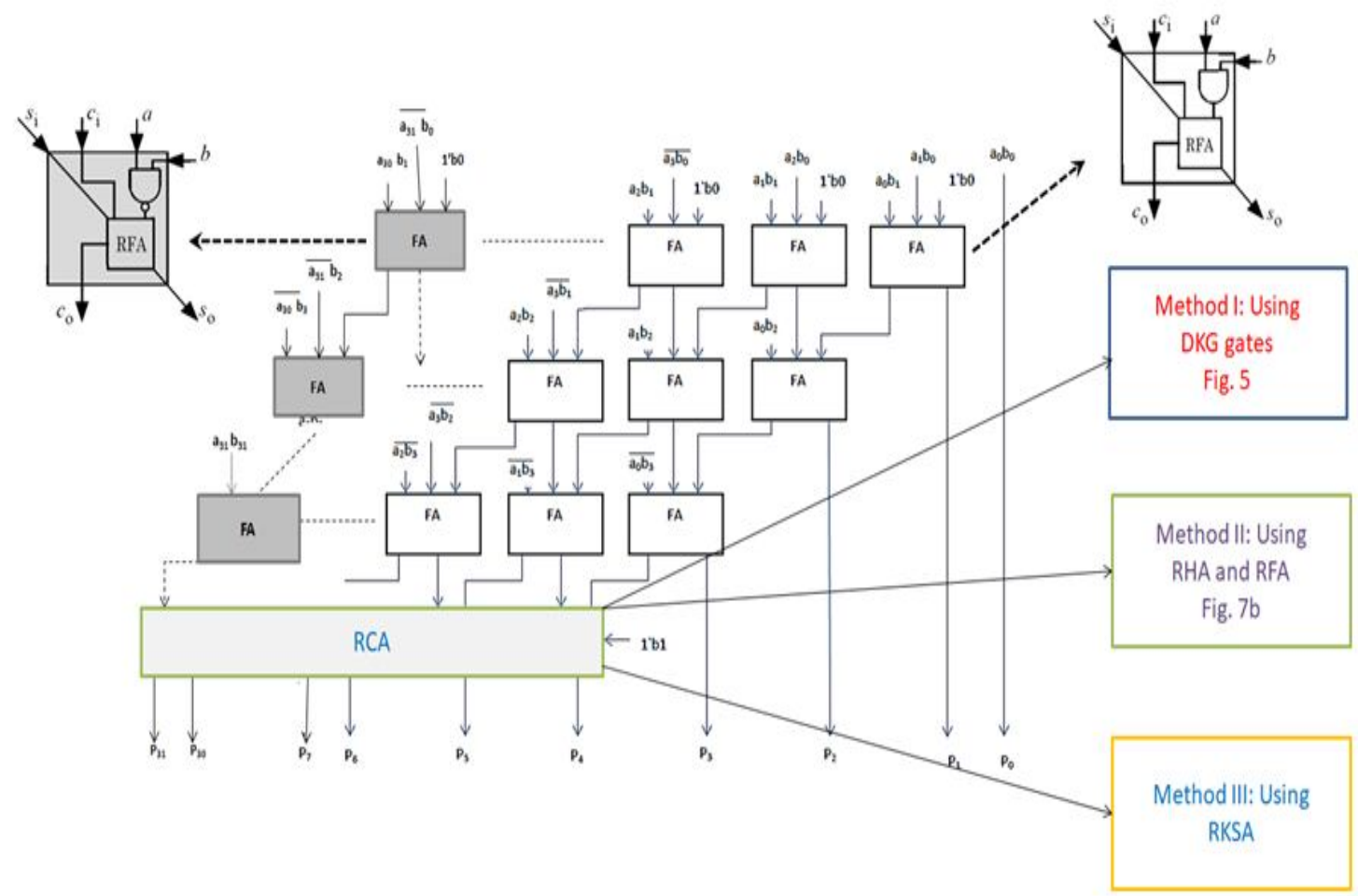

Figure. 9. Block diagram of $32 * 32 \mathrm{BWM}$ with proposed methods.
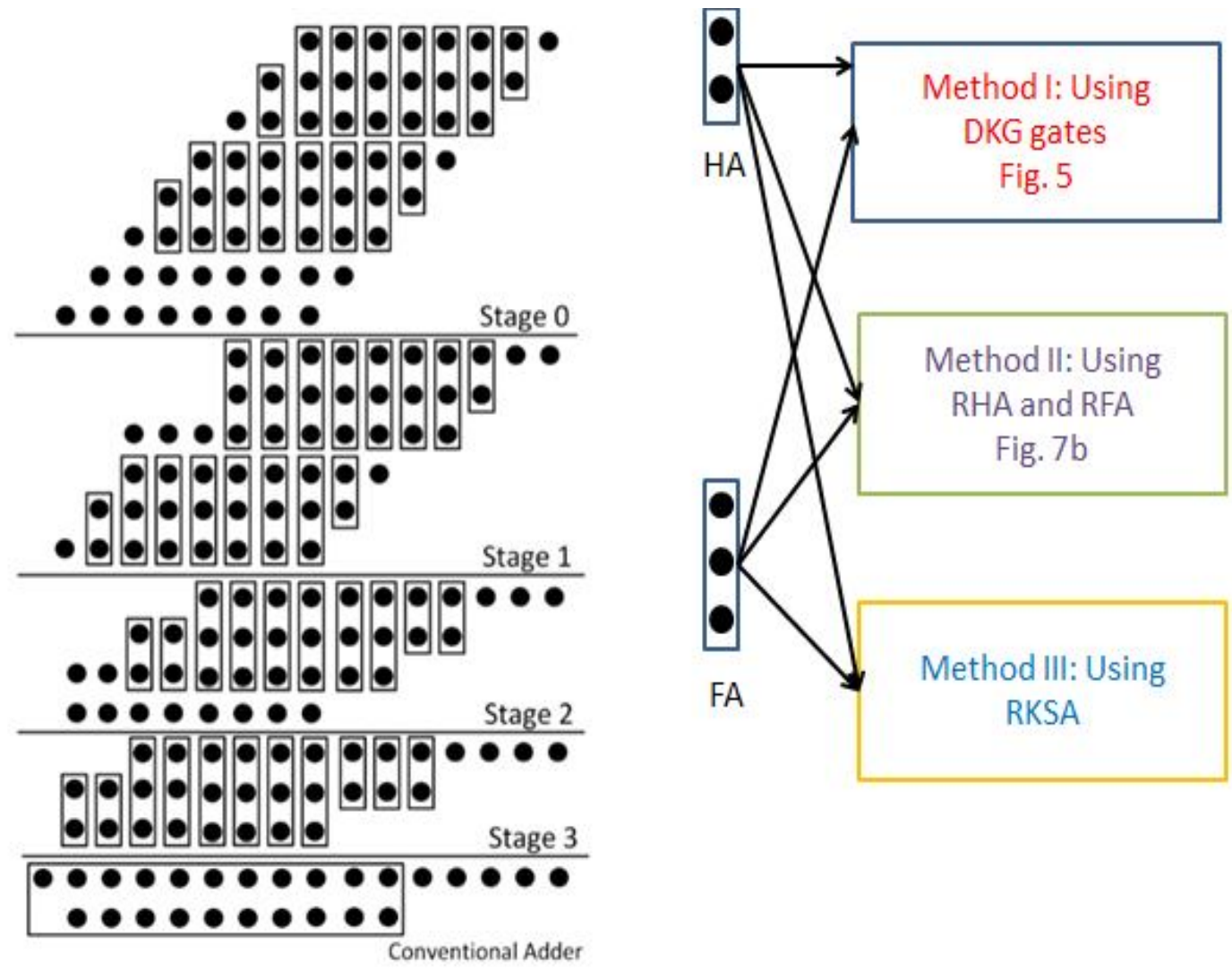

Figure. 10. Conventional Wallace Tree Multiplier. 
Anitha R et al., International Journal of Emerging Trends in Engineering Research, 8(10), October 2020, 7491 - 7500

Table 1: Comparison of Performance metrics of existing and proposed Designs

\begin{tabular}{|l|c|c|}
\hline \multicolumn{1}{|c|}{ Design } & Delay (ns) & Power (mW) \\
\hline Vedic Multiplier UT [29] & 35.76 & 166 \\
\hline Vedic Multiplier with DKG method 1 & 11.870 & 189.402 \\
\hline Vedic Multiplier with Proposed RHA and RFA method 2 & 8.161 & 182.37 \\
\hline Vedic Multiplier with Proposed RKSA method 3 & 7.770 & 170 \\
\hline Baugh Wooley with RCA [40] & 63.79 & 195.574 \\
\hline Baugh Wooley with proposed reversible logic 1(DKG) & 54.626 & 228.012 \\
\hline Baugh Wooley with proposed reversible logic 2 (proposed RHA and RFA) & 16.825 & 188.210 \\
\hline Proposed Baugh Wooley Multiplier with Reversible KSA & 11.863 & 171.019 \\
\hline Wallace Tree Multiplier with proposed reversible logic method 2 (proposed RHA & 9.421 & 168.23 \\
and RFA) & & 170.815 \\
\hline Wallace Tree with reversible logic proposed method 1 (DKG) & 10.761 & 196.016 \\
\hline Wallace Tree with reversible logic with RKSA & 10.294 & 15.876 \\
\hline Wallace tree multiplier [24] & & 182.911 \\
\hline
\end{tabular}


Anitha R et al., International Journal of Emerging Trends in Engineering Research, 8(10), October 2020, $7491-7500$

\section{FUTURE SCOPE}

The Vedic methods are efficient in reducing the delay and the power also. So the Vedic sutra can be implemented in several other multipliers and so many arithmetic operations to make the digital system very efficient. And in similar way reversible logic also plays a vital role in reducing the power. Several other designs of adders can be designed using reversible logic to get a better performance in different parameters.

\section{ACKNOWLEDGEMENT}

We acknowledge the support of Department of Micro and Nano Electronics, School of Electronics Engineering, Vellore Institute of Technology, Vellore.

\section{REFERENCES}

1. Y.-J. Jang, Y. Shin, M.-C. Hong, J.-K. Wee, S. Lee. Low-Power 32bit $\times$ 32bit Multiplier Design with Pipelined Block-Wise Shutdown: High Performance Computing - HiPC 2005, 12th International Conference, Goa, India, Springer, 3769, pp. 398-406, 2005

2. Z. Huang, High-level optimization techniques for low-power multiplier design, $\mathrm{Ph} . \mathrm{D}$. thesis; University of California, 2003

3. M. Ito, D. Chinnery, K. Keutzer. Low power multiplication algorithm for switching activity reduction through operand decomposition $21 \mathrm{st}$ International Conference on Computer Design pp. 21-26, 2003.

4. Landauer, R. Irreversibility and heat generation in the computing process. IBM journal of research and development, 5(3), 183-191, 1961

5. E. Jaya, K.C. Rao. Power, area and delay comparision of different multipliers, International Journal of Science, Engineering and Technology Research (IJSETR) Volume 5, Issue 6, June, 2016.

6. V.S. Kumar. Analysis of energy efficient PTL based full adders using different nanometer technologies Electronics and Communication Systems (ICECS), 2nd International Conference on, IEEE, 2015, pp. 310-315, 2015

7. A. Dubey, S. Akashe, S. Dubey. A novel high-performance CMOS 1 bit full-adder cell. Intelligent Systems and Control (ISCO), $7^{\text {th }}$ International Conference on, IEEE, 2013, pp. 312-315, 2013

8. S. Srikanth, I.T. Banu, G.V. Priya, G. Usha. Low power array multiplier using modified full adder. Engineering and Technology (ICETECH), 2016 IEEE International Conference on, IEEE, pp. 1041-1044, 2016.

9. S. Nair, A. Saraf, A Review Paper on Comparison of Multipliers based on Performance Parameters. ICSAT, 2014
10. J. Mitra, T. Nayak, Reconfigurable very high throughput low latency VLSI (FPGA) design architecture of CRC 32, "Intgr. VLSI J. 56, 1-14, 2017

11. Wang, J. Shield,. The efficient implementation of an array multiplier, Electro Information Technology, IEEE International Conference on, IEEE, p. 5, 2005

12. M.H. Ali, A.K. Sahani, Study, implementation and comparison of different multipliers based on array, KCM and vedic mathematics using EDA tools, Int. J. Sci. Res. Publ. 3 (6), 1, 2013

13. M. Sjalander, P. Larsson-Edefors, High-speed and low-power multipliers using the Baugh-Wooley algorithm and HPM reduction tree, Electronics, Circuits and Systems, ICECS 2008. 15th IEEE International Conference on, IEEE, pp. 33-36, 2008

14. K. Namba, I. Hideo, Design of defect tolerant wallace multiplier, "null, IEEE 300-304, 2005

15. K. Thilagavathi, S. Sivanantham, Two-stage low power test data compression for digital VLSI circuits, Computers \& Electrical Engineering 71, pp.309-320, 2018

16. Nielsen MA, Chuang IL, Quantum computation and quantum information, Cambridge University Press, New York, 2000

17. Vedral V, Barenco A, Ekert A, Quantum networks for elementary arithmetic operations, Phys Rev A 54(1), 147-153, 1996

18. Landauer, R. Irreversibility and Heat Generation in the Computing Process, IBM Journal of Research and Development,5,183-191. http://dx.doi.org/10.1147/rd.53.0183, 1961

19. Bennett, C.H. Logical Reversibility of Computation, IBM Journal of Research and Development, 17,525-532, 1973 http://dx.doi.org/10.1147/rd.176.0525

20. Bhagyalakshmi, H.R. and Venkatesha, M.K. An Improved Design of a Multiplier Using Reversible Logic Gates, International Journal on Engineering Science and Technology, 2, 3838-3845,2010.

21. Haghparast, M., Jassbi, S.J., Navi, K. and Hashemipour, O. Design of a Novel Reversible Multiplier Circuit Using HNG Gate in Nanotechnology, World Applied Sciences Journal, 3, 974-978, 2008

22. Islam, M.S., et al. Low Cost Quantum Realization of Reversible Multiplier Circuit, Information Technology Journal, 8,208-213, 2009 http://dx.doi.org/10.3923/itj.2009.208.213

23. Rangaraju, H.G., Babu Suresh, A. and Muralidhara, K.N. Design of Efficient Reversible Multiplier, Proceedings of the Second International Conference on Advances in Computing and Information Technology (ACITY), July 13-15, 2012

24. Akbar, E.P.A \& Mosleh, M.. An efficient design for reversible wallace unsigned multiplier. Theoretical Computer Science, 773, 43-52., 2019

25. Zhou, R., et al. Comment on Design of a Novel Reversible Multiplier Circuit using HNG Gate in 
Anitha R et al., International Journal of Emerging Trends in Engineering Research, 8(10), October 2020, 7491- 7500

Nanotechnology, World Applied Sciences Journal, 10, 161-165, 2010.

26. Vasudevan, D. P., Lala, P. K., Di, J., \& Parkerson, J. P. Reversible-logic design with online testability. IEEE transactions on instrumentation and measurement, 55(2), 406-414, 2006.

27. Garipelly, R., Kiran, P. M., \& Kumar, A. S. A review on reversible logic gates and their implementation. International Journal of Emerging Technology and Advanced Engineering, 3(3), 417-423, 2013.

28. A. Peres, Reversible logic and quantum computers, Physical Review A, Vol. 32, No. 6, pp: 3266-3276,1985.

29. Ramalatha, M.Dayalan, K D Dharani, P Priya, and S Deoborah, High Speed Energy Efficient ALU design using Vedic multiplication techniques, International Conference on Advances in Computational Tools for Engineering Applications, 2009. ACTEA '09.pp. 600 -3, Jul 15-17, 2009.

30. Sree Nivas A and Kayalvizhi N. Implementation of Power Efficient Vedic Multiplier. International Journal of Computer Applications 43(16):21-24, Published by Foundation of Computer Science, New York, USA, 2012

31. R.Bhaskar, Ganapathi Hegde, P.R.Vaya, An efficient hardware model for RSA Encryption system using Vedic mathematics, International Conference on Communication Technology and System Design 2011 Procedia Engineering 30,124 - 128, 2012

32. Mohanty, P, An Efficient Baugh-Wooley Architecture for Both Signed \& Unsigned Multiplication, International Journal of Computer Science \& Engineering Technology (IJCSET), 3, No., 2012

33. Lekshmi Viswanath, Ponni.M, Design and Analysis of 16 Bit Reversible ALU, IOS Journal of Computer Engineering (IOSRJCE) ISSN : 2278-0661 Volume 1, Issue 1, PP 46-53,2012.

34. Wallace, C. S. A suggestion for a fast multiplier. IEEE Transactions on electronic Computers, (1), 14-17, 1964.

35. Peres, A.. Reversible logic and quantum computers. Physical review A, 32(6), 3266, 1985.

36. Rajasree Shanmuganathana , Kathirvel Brindhadevi, Comparative analysis of various types of multipliers for effective low power, Microelectronic Engineering, Volume 214, 1, Pages 28-37, 2019. https://doi.org/10.1016/j.mee.2019.04.015.

37. Akbar, E.P.A., Haghparast, M., Navi, K. Novel design of a fast reversible Wallace sign multiplier circuit in nanotechnology. Microelectron. J. 42(8), 973-981, 2011.

38. Kokila Bharti Jaiswal, Nithish Kumar V, Pavithra Seshadri and Lakshminarayanan G, Low Power Wallace Tree Multiplier Using Modified Full Adder, International Conference on Signal Processing, Communication and Networking (ICSCN), 2015.

39. K. A. C. Bickerstaff, M. Schulte, E. E. Swartzlander, Reduced area multipliers, International Conference on Application-Specific Array Processors, Venice, 25-27, pp.478-489, 1993. 